\title{
HSC/12/05
}

\section{A new method for automated noise cancellation in electromagnetic field measurement}

\author{
Paweł Bieńkowski* \\ Krzysztof Burnecki** \\ Joanna Janczura** \\ Rafał Weron*** \\ Bartłomiej Zubrzak*
}

* Institute of Telecommunications, Teleinformatics and Acoustics, Wrocław University of Technology, Poland

** Hugo Steinhaus Center, Wrocław University of Technology, Poland

*** Institute of Organization and Management, Wrocław University of Technology, Poland

Hugo Steinhaus Center Wrocław University of Technology

Wyb. Wyspiańskiego 27, 50-370 Wrocław, Poland http://www.im.pwr.wroc.pl/ hugo/ 


\title{
A NEW METHOD FOR AUTOMATED NOISE CANCELLATION IN ELECTROMAGNETIC FIELD MEASUREMENT
}

\author{
P. Bieńkowski ${ }^{1, *}$, K. Burnecki ${ }^{2}$, J. Janczura ${ }^{2}$, R. Weron ${ }^{3}$, \\ B. Zubrzak ${ }^{1}$
}

1 Institute of Telecommunications, Teleinformatics and Acoustics, Wrocław University of Technology, 50-370 Wrocław, Poland

2 Hugo Steinhaus Center, Institute of Mathematics and Computer Science, Wrocław University of Technology, 50-370 Wrocław, Poland

${ }^{3}$ Institute of Organization and Management, Wrocław University of Technology, 50-370 Wrocław, Poland

\begin{abstract}
Electromagnetic field (EMF) measurements have limited accuracy, which is additionally impaired by meter self-noise influence. In this paper a novel noise cancellation method is proposed, based on the Hidden Markov Model (HMM) methodology. It allows to calculate the overall field intensity with a much higher accuracy than that obtained from other popular approaches, especially when EMF measurements are close to the noise level. The effectiveness of the new method is illustrated on two EMF datasets, one recorded in an urban and another in a rural area. Its performance is further evaluated in a thorough simulation study using datasets representing the two distinct noisy environments.
\end{abstract}

\section{INTRODUCTION}

The progress of civilization is marked by intensive growth of emissions to the environment. The intensification of the electromagnetic field $(\mathrm{EMF})$ is a result of increasing energy demand (more and more devices are powered by electric energy) and the popularization of radio communication systems, especially data transmission. In the past, the dominant EMF sources of broadcasting systems used to be radio and TV transmitters, usually located at dedicated sites. In modern duplex

* Corresponding author: P. Bieńkowski (pawel.bienkowski@pwr.wroc.pl). 
systems, however, EMF sources can be found both in dense networks of base stations and within mobile terminals. The latter are usually low power sources, but exist in large quantity. Despite the fact that in most cases the measured EMF intensity is well below hazardous levels, there is a need for constant and preferably automated monitoring of this exposure with the ultimate objective of predicting alarming trends that could threaten the society [2].

To this end, we develop a novel noise cancellation method based on the Hidden Markov Model (HMM) methodology. HMMs have been exploited in many areas including pattern recognition, seismic radiation and electricity markets $[3,6]$. In this paper we show that HMMs can serve as a useful tool for the decomposition of EMF measurements into the "real" signal and noise. In Section 2 we briefly review the methodology of EMF measurement. In the following Section we recall commonly used methods for minimizing self-noise influence on measurements and introduce our method. Next, in Section 4 we apply these methods to two EMF datasets, one recorded in an urban and another in a rural area. We also evaluate the performance of the HMM method in a thorough simulation study using datasets representing the two distinct noisy environments. Finally, in Section 5 we conclude.

\section{EMF MEASUREMENT METHODOLOGY}

The electromagnetic field is characterized by a number of parameters. However, when considering the influence of EMF on the biosphere, the most important information is the intensity of particular EMF elements in defined frequency ranges. By analyzing frequency bands, it is possible to identify the EMF source class, for example, in radio communication: radio diffusion and mobile radio communication, etc. This approach prefers broadband measurements or single band measurements using meters with passive broadband probes, which allow measurements in direct surroundings of primary and secondary EMF sources. Broadband meters are widely used in measurements related to work safety and environment protection. Since the meter manufacturers have developed spherical and frequency characteristic shaping of the probes, the resulting measurements are equivalent to field intensity from the entire probe frequency range. The elementary weakness of this technique is a relatively high detection threshold, limited with detector sensitivity to a minimum of about $1 \mathrm{~V} / \mathrm{m}$ (up to $0.1 \mathrm{~V} / \mathrm{m}$ in the individual projects) and measurement dynamics less than $40 \mathrm{~dB}$. These values can be found in manufacturer specifications of most of the common broadband EMF meters. Our measurements show that the self-noise level is oscillating between 0.12 and $0.35 \mathrm{~V} / \mathrm{m}$ 
for more sensitive and between 0.8 and $1.7 \mathrm{~V} / \mathrm{m}$ for less sensitive probes. These values can increase in the course of time, what can be explained by electric circuit aging and probe characteristic change related to momentary probe overrides. As a result, even in the case of "electromagnetic silence", the average meter indication will take values above mentioned levels. Among other factors limiting EMF measurement accuracy (regardless of the method) let us mention the field distribution around the EMF sources.

In Figure 1 we compare measurement results obtained from three common EMF meters. Measurements were made by putting the devices in a standard EMF with known intensity value ( $X$ axis) and recording 50 measurement results. We can observe a dispersion of results which illustrates meter indication instability and influence of self-noises on measurement results. As expected, with the growth of EMF intensity, the results are more and more repetitive and better match the actual EMF value $\left(E_{\text {standard }}\right)$. Moreover, even in the case of "electromagnetic silence" the average meter indication will take a value corresponding to its self-noise level. This can cause false instrument readings when measuring EMF level trends, if the measured EMF levels are lower than the meter self-noise.

A much lower detection threshold and significantly greater dynamics can be reached using the selective measurement technique preferred in EMF exposure measurements at GSM base stations [1, 12. It is recommended to use in such systems specially designed multiband 5] or wideband antennas [8, which allow to obtain sensitivity at the level of a single $\mathrm{mV} / \mathrm{m}$ with dynamics up to $140 \mathrm{~dB}$, and to apply bandpass filters to minimize noise influence [9]. The resulting field intensity $E_{\text {eval }}$ corresponding to a broadband measurement is calculated using

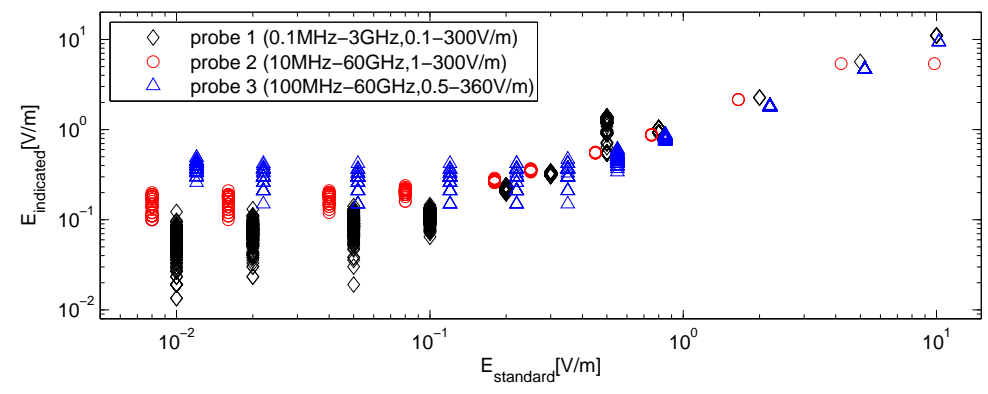

Figure 1. Self-noise measurement of common EMF meters. 
the following formula:

$$
E_{\text {eval }}=\sqrt{\sum_{n} E_{n}^{2}}
$$

where $E_{n}$ is the EMF intensity from band $n$ with width corresponding to the RBW filter used in spectrum analyzer measurements.

In this case we also have to deal with the meter self-noise, especially when analyzing EMF intensity from the entire meter frequency range and relatively low measured EMF levels. In such a situation, components affected by the meter self-noise become much more meaningful in the resultant EMF intensity. Based on authors' rough estimates, in the case of measurements, where EMF intensity exceeds slightly the noise level, the errors can even reach a value of 10 $\mathrm{dB}$. It is desirable to minimize this background noise influence [11.

\section{METHODS OF MINIMIZATION OF SELF-NOISE INFLUENCE ON MEASUREMENT RESULT}

\subsection{Commonly used methods}

The simplest method of noise elimination is cutting off (or zeroing) all the spectrum components whose level does not exceed a given value. It is useful only when the noise level is constant, not related to frequency and possible to obtain using only selective EMF meters. Another method is the average noise level measurement, which is constructed by subtracting the meter noise level from each sample $E_{n}$. In this method formula (11) takes the form:

$$
E_{\text {eval-noise }}=\sqrt{\sum_{n}\left(E_{n}-E_{n, \text { noise }}\right)^{2}},
$$

where $E_{n, n o i s e}$ is the meter noise level for the frequency corresponding to the $n$-th sample of the signal. This method is applicable when the meter self-noise is known and independent of both time and meter settings. Using this approach it is possible to decrease the noise level influence by a few $\mathrm{dB}$.

A much better method, applicable in any conditions, is to take into account only those sub-bands from the whole spectrum, which contain useful signals. This can be done by selecting the signal peaks from the noise background. In practice, when performing measurement using a selective EMF meter, the operator chooses which signal is treated as potentially significant and taken as a component of the EMF intensity value. The problem with this approach is that it is subjective - it heavily depends on the meter operator's experience and perception of 
what is a signal and what is not. It is exactly our aim to automate the frequency sub-band selection process and make it more objective and meter operator-independent.

\subsection{A new method based on HMM filtering}

The underlying idea behind Hidden Markov Models (HMMs) is to describe the observed phenomena by two (or more) separate phases or regimes with different statistical properties. Since the observed EMF at each frequency band $n$ can be either the meter noise or the "real" EMF, we use a two state HMM. The measurements $E_{n}$ are described by two separate states (phases, regimes) with different underlying properties. The values $E_{n, i}$ within a given regime are assumed to be independent, identically distributed random variables following some distribution function $F_{i}$. Here, we use the Gaussian distribution for the noise and the log-normal distribution for the "real EMF" regime:

$$
E_{n}= \begin{cases}E_{n, 1} \sim \mathrm{N}\left(\mu_{1}, \sigma_{1}^{2}\right) & \text { if } R_{n}=1, \\ E_{n, 2} \sim \mathrm{LN}\left(\mu_{2}, \sigma_{2}^{2}\right) & \text { if } R_{n}=2,\end{cases}
$$

where "1" is the noise regime, "2" denotes the "real" EMF regime and $R_{n}$ is the state variable for band $n$. Recall, that the random variable $X$ is driven by the log-normal distribution with parameters $\mu$ and $\sigma$ if $\ln (X) \sim \mathrm{N}\left(\mu, \sigma^{2}\right)$. Such a choice of distributions is motivated by a good fit to the analyzed data. However, nothing prevents us from using other probability laws in the HMM approach.

Definition (3) implies that the statistical properties of the measurements differ depending on the actual state $R_{n}$. The switching mechanism between the states is assumed to be governed by an unobserved (latent) Markov chain. Consequently, we have a probability law that governs the transition from one state to another. It is given by the transition matrix $\mathbf{P}$ which contains the probabilities $p_{i j}=P\left(R_{n+1}=j \mid R_{n}=i\right)$ of switching from regime $i$ to regime $j$, when changing from frequency band $n$ to $n+1$.

HMM identification (regimes, parameters) can be performed in a number of ways. Here we follow [7] and apply the expectationmaximization (EM) algorithm, where the whole set of parameters $\theta$ is estimated within an iterative two-step procedure. The algorithm starts with an arbitrarily chosen vector of initial parameters $\theta^{(0)}=$ $\left(\mu_{i}^{(0)}, \sigma_{i}^{(0)}, \mathbf{P}^{(0)}, \rho_{i}^{(0)}\right)$, for $i=1,2$, where $\rho_{i}^{(0)} \equiv P\left(R_{1}=i\right)$. In the first step of this iterative procedure (the E-step) inferences about the state values, i.e., the conditional probabilities $P\left(R_{n}=i \mid E_{1}, \ldots, E_{N} ; \theta\right)$ for the measurement being in regime $i$ at frequency band $n$, are made. Next, in the second step (the M-step) new maximum likelihood (ML) estimates 
3 of the parameter vector $\theta$, based on the smoothed inferences obtained in the E-step, are calculated. Both steps are repeated until the (local) maximum of the likelihood function is reached. A detailed description of the algorithm is given below.

E-step: Assume that $\theta^{(k)}$ is the parameter vector calculated in the M-step during the previous iteration. The E-part consists of the following steps [6, 7]:

i) Forward filtering: for $n=1,2, \ldots, N$ iterate on equations:

$$
P\left(R_{n}=i \mid \mathbf{E}_{n} ; \theta^{(k)}\right)=\frac{P\left(R_{n}=i \mid \mathbf{E}_{n-1} ; \theta^{(k)}\right) g_{i}\left(E_{n} ; \theta^{(k)}\right)}{\sum_{i=1}^{2} P\left(R_{n}=i \mid \mathbf{E}_{n-1} ; \theta^{(k)}\right) g_{i}\left(E_{n} ; \theta^{(k)}\right)},
$$

where $\mathbf{E}_{n}=\left(E_{1}, E_{2}, \ldots, E_{n}\right)$ is the vector of measurements and $g_{i}\left(E_{n} ; \theta^{(k)}\right)$ is the probability density function (PDF) of the EMF at frequency band $n$ conditional on the measured value coming from regime $i$ (here, $g_{1}$ and $g_{2}$ are Gaussian and log-normal PDFs, respectively); and

$$
P\left(R_{n+1}=i \mid \mathbf{E}_{n} ; \theta^{(k)}\right)=\sum_{j=1}^{2} p_{j i}^{(k)} P\left(R_{n}=j \mid \mathbf{E}_{n} ; \theta^{(k)}\right),
$$

until $P\left(R_{N}=i \mid \mathbf{E}_{N} ; \theta^{(k)}\right)$ is calculated. The starting point for the iteration is: $P\left(R_{1}=i \mid \mathbf{E}_{0} ; \theta^{(k)}\right)=\rho_{i}^{(k)}$.

ii) Backward filtering: for $n=N-1, N-2, \ldots, 1$ iterate on

$P\left(R_{n}=i \mid \mathbf{E}_{N} ; \theta^{(k)}\right)=\sum_{j=1}^{2} \frac{P\left(R_{n}=i \mid \mathbf{E}_{n} ; \theta^{(k)}\right) P\left(R_{n+1}=j \mid \mathbf{E}_{N} ; \theta^{(k)}\right) p_{i j}^{(k)}}{P\left(R_{n+1}=j \mid \mathbf{E}_{n} ; \theta^{(k)}\right)}$.

M-step: The new parameter estimates $\theta^{(k+1)}$ can be derived by maximizing the log-likelihood function. In the considered case:

$$
\begin{gathered}
\mu_{i}^{(k+1)}=\frac{\sum_{n=1}^{N} f_{i, n} P\left(R_{n}=i \mid \mathbf{E}_{N} ; \theta^{(k)}\right)}{\sum_{n=1}^{N} P\left(R_{n}=i \mid \mathbf{E}_{N} ; \theta^{(k)}\right)}, \\
\left(\sigma_{i}^{(k+1)}\right)^{2}=\frac{\sum_{n=1}^{N} g_{i, n} P\left(R_{n}=i \mid \mathbf{E}_{N} ; \theta^{(k)}\right)}{\sum_{n=1}^{N} P\left(R_{n}=i \mid \mathbf{E}_{N} ; \theta^{(k)}\right)},
\end{gathered}
$$

where $f_{1, n}=E_{n}, f_{2, n}=\mathbb{I}_{\left\{E_{n}>0\right\}} \ln \left(E_{n}\right)$, II is the indicator function, $g_{1, n}=\left[E_{n}-\mu_{1}^{(k+1)}\right]^{2}, g_{2, n}=\left[\ln \left(E_{n}\right)-\mu_{2}^{(k+1)}\right]^{2}$ and $i=1,2$. 
Finally, we have $\rho_{i}^{(k+1)}=P\left(R_{1}=i \mid \mathbf{E}_{N} ; \theta^{(k)}\right)$ and the transition probabilities are estimated according to the following formula [6, 7]:

$$
p_{i j}^{(k+1)}=\frac{\sum_{n=2}^{N} P\left(R_{n}=j \mid \mathbf{E}_{N} ; \theta^{(k)}\right) \frac{p_{i j}^{(k)} P\left(R_{n-1}=i \mid \mathbf{E}_{n-1} ; \theta^{(k)}\right)}{P\left(R_{n}=j \mid \mathbf{E}_{n-1} ; \theta^{(k)}\right)}}{\sum_{n=2}^{N} P\left(R_{n-1}=i \mid \mathbf{E}_{N} ; \theta^{(k)}\right)},
$$

where $p_{i j}^{(k)}$ is the transition probability from the previous iteration. All values obtained in the M-step are then used as a new parameter vector $\theta^{(k+1)}=\left(\mu_{i}^{(k+1)}, \sigma_{i}^{(k+1)}, \mathbf{P}^{(k+1)}, \rho_{i}^{(k+1)}\right), i=1,2$, in the next iteration of the E-step. The algorithm is terminated when $\left|\theta^{(k)}-\theta^{(k-1)}\right|<\delta$ for some small $\delta$.

\section{EMPIRICAL RESULTS}

\subsection{EMF datasets and data preprocessing}

We illustrate the effectiveness of the HMM-based method using EMF intensity measurements obtained with a selective meter SRM-3000 sn. M-0008 with an isotropic probe (band $75 \mathrm{MHz}-3 \mathrm{GHz}$ ) sn. H-0185. Both were calibrated in a certified calibrating laboratory (calibration certificate no. LWIMP/W/009/10). The datasets were collected in an urban (Wrocław downtown - D1) and a rural (a small village in the Sudety Mountains - D2) area to obtain spectra with more and less significant spectral bands. As can be observed in the top panels of Figure 2, the noise is non-homogeneous. The noise level changes with frequency and the variance of the noise increases with noise level (note, that in Figure 2 the variance seems to be stable only because a logarithmic scale is used). Consequently, in order to deal with the variance dependence on the noise level, we worked with the logarithm of the signal (and not the signal itself). To cope with the changing noise level we estimated it using a moving median of length $N / 10$, where $N$ is the number of frequency bands in the dataset.

\subsection{HMM filtering results}

We fitted a HMM defined in Section 3.2 to the transformed datasets, i.e., to $X(n)=\log \left(E_{n}\right)-\log \left(L_{n o i s e}\right)$, where $L_{\text {noise }}$ stands for the "moving median"-estimated noise level. The filtering results for the transformed values $X(n)$, i.e., the probabilities $P\left(R_{n}=2 \mid \mathbf{E}_{N}\right)$ of being in the "real" EMF regime (conditional on the information included in the whole dataset $\mathbf{E}_{N}$ ), are plotted in the bottom panels of Figure 2 . 

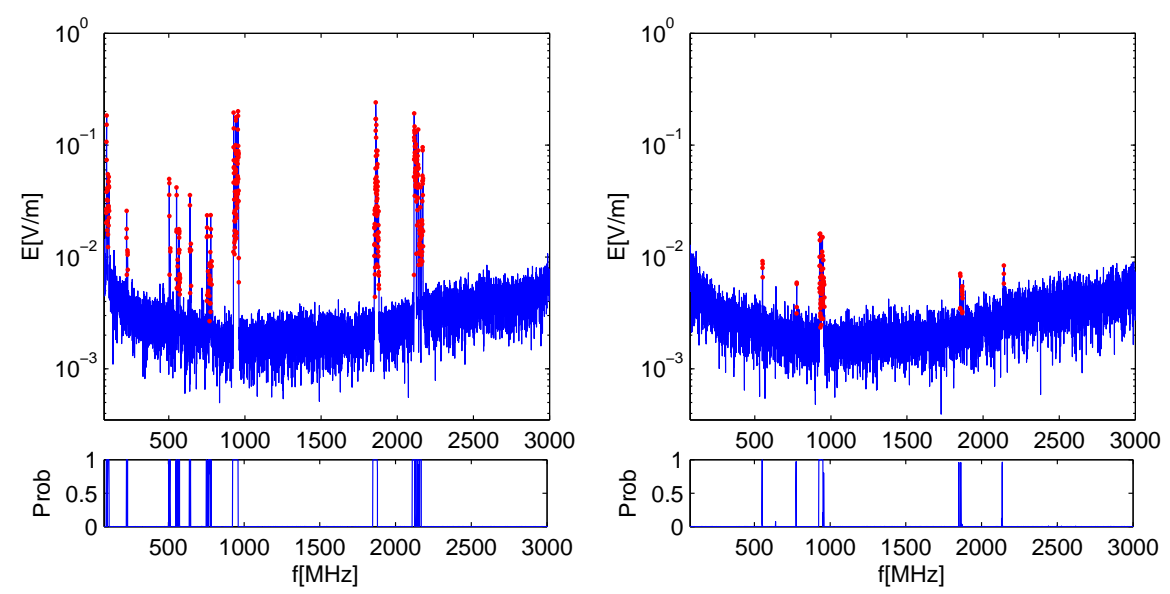

Figure 2. Filtering results for datasets D1 (a) and D2 (b). The probabilities $P\left(R_{n}=2 \mid \mathbf{E}_{N}\right)$ of being in the "real" EMF regime are plotted in the bottom panels. Values classified into the "real" EMF regime, i.e., with $P\left(R_{n}=2 \mid \mathbf{E}_{N}\right)>0.5$ are denoted by dots in the top panels depicting the original signal $E_{n}$ on a logarithmic scale.

Table 1. EMF intensity values calculated using three different noise reduction methods.

\begin{tabular}{cccc}
\hline \hline Data & $E_{\text {eval }}[\mathrm{V} / \mathrm{m}]$ & $E_{\text {eval-noise }}[\mathrm{V} / \mathrm{m}]$ & $E_{H M M}[\mathrm{~V} / \mathrm{m}]$ \\
\hline $\mathrm{D} 1$ & 1.0906 & 1.0319 & 1.0647 \\
$\mathrm{D} 2$ & 0.2505 & 0.0873 & 0.0670 \\
\hline \hline
\end{tabular}

In our procedure the values with $P\left(R_{n}=2 \mid \mathbf{E}_{N}\right)>0.5$ are classified as belonging to the "real" EMF regime and denoted by dots in the top panels. This classification allows for automated selection of those frequency sub-bands from the whole spectrum, which contain useful signals, i.e., the "real" EMF signal. Summing $E_{n}^{2}$ only over those subbands yields the HMM estimate, $E_{H M M}$, of the field intensity.

For comparison purposes, we calculated the field intensity values using three different approaches, see Table 1. The $E_{\text {eval }}$ values were calculated from (1) for the whole dataset (D1 or D2) with unreduced meter noise, the $E_{\text {eval-noise }}$ values were derived using the average noise level method (2) with a "moving median"-estimated noise level, and the $E_{H M M}$ values were obtained by applying the HMM methodology. We can observe that the differences between the three approaches are 
most pronounced for the D2 dataset, i.e., for a signal which is close to the noise level. In the following Section we will demonstrate in a simulation study that these results are significant and that the HMM approach yields very accurate field intensity measurements.

\subsection{Validation of the HMM-based methodology}

In order to check the accuracy of the method we performed Monte Carlo simulations [3, 10, 13] using two distinct noisy environments: one representing an urban (dataset D1) and another a rural area (D2). In each of the two scenarios we simulated 1000 EMF samples of the same length as the original datasets. The simulated samples consisted of two parts: (i) self-noise level of the meter and (ii) EMF signals generated from a log-normal distribution, with parameters estimated within the HMM identification procedure, added to the noise.

Next, like in Section 4.2, we calculated the field intensity values using three different approaches to noise reduction: $E_{\text {eval }}, E_{\text {eval-noise }}$ and $E_{H M M}$. Having the EMF intensities, we computed the respective mean absolute percentage errors (MAPEs) [4, 13, see Table 2. The errors were evaluated with respect to the simulated signals, for which we knew the frequency bands with the "real" EMF signals. The HMM results are extremely accurate, which is most visible for dataset D2 where signal to noise ratio is low. Obviously, completely ignoring the noise leads to totally unacceptable errors of the field measurement.

Apart from the analysis of the field intensity, we also checked the efficiency of the HMM method in classification of the measurements into "real" EMF signal and noise. Three indicators of the performance (or quality) of the methods are reported in Table 2. The first concerns the proportion of the number of successfully identified signals to the overall number of simulated signals (sensitivity), the second is the proportion of the number of successfully identified noise values to the

Table 2. Mean absolute percentage errors (MAPE) of the EMF intensity values calculated using three different approaches to noise reduction, as measured for 1000 simulated samples reproducing each of the two environments (D1 and D2). Performance measures (sensitivity, specificity and precision; see text for details) for the HMM method are also reported.

\begin{tabular}{ccccccc}
\hline \hline Data & & MAPE [\%] & \multicolumn{3}{c}{ Performance [\%] } \\
type & $E_{\text {eval }}$ & $E_{\text {eval-noise }}$ & $E_{H M M}$ & Sens. & Spec. & Prec. \\
\hline D1 & 0.02 & 0.10 & 0 & 99.99 & 100 & 100 \\
D2 & 154.16 & 9.80 & 0.05 & 99.54 & 99.99 & 100 \\
\hline \hline
\end{tabular}


overall number of simulated noise values (specificity), and the third is the proportion of the number of successfully identified signals to the overall number of identified signals (precision) [13. Therefore, the first and second numbers describe the quality of identifying the real signals and noise values respectively, while the third stands for the quality of identified signals. They should be close to $100 \%$. We can observe that the HMM method works very well, since all indicators are above $99 \%$.

\section{CONCLUSIONS}

In this paper we presented a new method of minimization of self-noise influence on EMF measurements based on the HMM methodology. The most significant advantage of the introduced method is a substantial increase of measurement accuracy for values close to the noise level. The method can extract real EMF intensity from the background noise very well, which was illustrated on two distinct datasets and validated by means of Monte Carlo simulations. Such an improvement in the estimation of real EMF intensity could be very useful in tracking trends of the EMF level in the environment. The identification algorithm is fast and allows real-time post-processing in measuring devices. The whole procedure can be fully automated. It can be widely used when estimating the EMF intensity and its changes in time, especially in long-term environmental monitoring measurements, where EMF levels are close to the meter self-noise, when the elimination of its influence is very important. The presented method could be also a useful tool for verification of numerical simulations of radio signal propagations [14], where noise influence can significantly affect the simulation results.

\section{Acknowledgements}

This research was supported by grant no. POIG.01.03.01-02-002/08. Matlab codes for estimating EMF intensity using HMM filtering are available from IDEAS: http://ideas.repec.org/s/wuu/hscode.html.

\section{REFERENCES}

1. Bieńkowski, P., H. Trzaska and B. Zubrzak, "Methods of the electromagnetic fields intensity level assessment and monitoring in the environment", 21st International Conference Radioelektronika, 259-262, 2011.

2. Canbay, C., "The essential environmental cause of multiple sclerosis disease", Progress In Electromagnetics Research, Vol. 101, 375-391, 2010. 
3. Cappe, O., E. Moulines and T. Ryden, Inference in Hidden Markov Models, Springer, 2005.

4. Gurel, C. S., H. Cosar, and O. Akalin, "Accurate resonant frequency computation of multisegment rectangular dielectric resonator antennas", Journal of Electromagnetic Waves and Applications, Vol. 24, No. 5/6, 839-847, 2010.

5. Han, Y., Y.-Z. Yin, Y.-Q. Wei, Y. Zhao, B. Li, and X. N. Li, "A novel triple-band monopole antenna with double coupled c-shaped strips for wlan/wimax applications", Journal of Electromagnetic Waves and Applications, Vol. 25, No. 8/9, 1308-1316, 2011.

6. Janczura, J. and R. Weron, "Efficient estimation of Markov regime-switching models: An application to electricity wholesale market prices", AStA Adv. Stat. Anal., forthcoming. Online First DOI: 10.1007/s10182-011-0181-2, 2011.

7. Kim, C.-J., "Dynamic linear models with Markov-switching", Journal of Econometrics, Vol. 60, 1-22, 1994.

8. Lin, S.-C., T.-L. Jong, C.-W. Hsieh, and K.-J. Ho, "Wideband series-fed dipole antenna with balun integrated", Journal of Electromagnetic Waves and Applications, Vol. 24, No. 17/18, 2463 - 2477, 2010.

9. Liu, B., F. Wei, Q.-Y. Wu, and X.-W. Shi, "A tunable bandpass filter with constant absolute bandwidth", Journal of Electromagnetic Waves and Applications, Vol. 25, No. 11/12, 1596-1604, 2011.

10. Mineo, M., A. Di Carlo, and C. Paoloni, "Analytical design method for corrugated rectangular waveguide SWS THz vacuum tubes", Journal of Electromagnetic Waves and Applications, Vol. 24, No. 17/18, 2479-2494, 2010.

11. Orimoto, H. and A. Ikuta, "Signal processing for noise cancellation in actual electromagnetic environment", Progress In Electromagnetics Research, Vol. 99, 307-322, 2009.

12. Riederer, M. and G. Neubauer, "Measuring the low power, instantaneous urban field strengths due to GSM base stations", International Zurich Symposium on EMC, 266-270, 2003.

13. Sammut, C. and G. I. Webb (Eds), Encyclopedia of Machine Learning, Springer, New York, 2011.

14. Staniec, K. and A. J. Pomianek, "On simulating the radio signal propagation in the reverberation chamber with the ray launching method", Progress in Electromagnetics Research B, Vol. 27, 83-99, 2011. 


\section{HSC Research Report Series 2012}

For a complete list please visit http://ideas.repec.org/s/wuu/wpaper.html

01 Inference for Markov-regime switching models of electricity spot prices by Joanna Janczura and Rafał Weron

02 The relationship between spot and futures $\mathrm{CO} 2$ emission allowance prices in the EU-ETS by Stefan Trück, Wolfgang Härdle and Rafał Weron

03 Modeling of short term interest rate based on tempered fractional Langevin equation by Janusz Gajda

04 Anomalous dynamics of Black-Scholes model time-changed by inverse subordinators by Marcin Magdziarz and Janusz Gajda

05 A new method for automated noise cancellation in electromagnetic field measurement by Paweł Bieńkowski et al. 